\title{
Temporal Coherence Changes of Typical Urban Features Based on Sentinel-1A Data
}

\author{
Jiaojie Li, Xuedong Zhang, ${ }^{*}$ and Xianglei Liu** \\ Key Laboratory for Urban Geomatics of National Administration of Surveying, Mapping and Geoinformation, \\ Beijing University of Civil Engineering and Architecture, 1 Zhanlanguan Road, Beijing 100044, P. R. China
}

(Received September 30, 2020; accepted December 10, 2020)

Keywords: InSAR, Sentinel-1A, coherence, time scale

Using the principle of radar interferometry, we analyzed the temporal coherence changes of five typical ground features (residential areas, vegetation, bare soil, bridges, and factories and warehouses) in urban areas of Beijing using 29 images from Sentinel-1A equipped with a C-band synthetic aperture radar (SAR) sensor over one year. The results of the study showed the following. (1) Among the five typical ground features, the coherence of vegetation was the lowest. Owing to changes in its state and atmospheric conditions, the coherence of vegetation fluctuated sharply over the year. The coherence of factories and warehouses was the highest and relatively stable over the year. (2) Classifying the five typical ground features into artificial and natural features, we found that the artificial features of factories and warehouses, residential areas, and bridges maintained a high degree of coherence over the year. Among them, the coherence of residential areas was the most stable. The natural features of vegetation and bare soil were affected by the changes in their states and atmospheric conditions over the year. The research results can be used for the classification of land use types, the statistical analysis of urban green coverage, and the extraction of points with high coherence in long-term surface deformation inversion.

\section{Introduction}

In recent years, with the improvement of sensor technology and the quality of sensing data, remote sensing has become an important technique for obtaining geospatial information. Remote sensing can not only rapidly and synchronously acquire spatial data over a large area and measure and produce topographic maps of various scales and other spatial information products, but also be used for digital elevation model (DEM) production, ${ }^{(1-3)}$ surface deformation inversion, ${ }^{(4-7)}$ land use type classification, ${ }^{(8-10)}$ forest biomass mapping, ${ }^{(11,12)}$ and terrain slope information extraction. ${ }^{(13)}$ Recently, higher requirements have been placed on the acquisition of remote sensing information and information-processing technology. Interferometric synthetic aperture radar (InSAR) technology is a new technology that emerged in this context. ${ }^{(14)}$ As one of the most promising research fields in microwave remote sensing, InSAR can penetrate clouds, fog, smoke, and dust, enabling the all-weather and large-scale

\footnotetext{
*Corresponding author: e-mail: zhangxuedong@bucea.edu.cn

${ }^{* *}$ Corresponding author: e-mail: liuxianglei@bucea.edu.cn

https://doi.org/10.18494/SAM.2020.3136
} 
acquisition of surface information, especially in cloudy and rainy areas, ${ }^{(15)}$ where it is difficult for traditional optical sensors to image. Therefore, SAR images have become very important sources of remote sensing data.

Coherence is one of the key factors in ensuring the imaging performance of SAR data. Different ground features have different coherence values. ${ }^{(16)}$ Regarding the coherence of SAR, Ichoku et al., ${ }^{(17)}$ Andre and Morrison, ${ }^{(18)}$ and Lv et al. ${ }^{(19)}$ analyzed the factors affecting coherence and improved the coherence of different ground objects from the perspective of SAR system design and SAR image processing algorithm design. Anura et al. ${ }^{(20)}$ analyzed the seasonal variation in the coherence of SAR interferograms in Kiruna, northern Sweden. As one of the many specific applications of SAR image coherence, the extraction of urban changes is a hot research field. Chang et al. ${ }^{(21)}$ used a highly coherent pointy target of an urban area to extract the urban boundary. Zhang et al. ${ }^{(22)}$ combined the difference method and the coherence coefficient method to analyze the changes in an urban area, and verified the detection accuracy of the proposed method. Pulvirenti et al. ${ }^{(23)}$ theoretically discussed the advantages of combining intensity and coherence, and used SAR image coherence information for flood mapping in urban environments, which was verified with a flood case in Emilia-Romagna, northern Italy. The results showed that the multitime trend analysis of coherence can greatly reduce the extraction error of a flood-inundated area caused by only considering the intensity data. However, it is difficult to develop a flood mapping algorithm with the SAR coherence coefficient. Wang et al. ${ }^{(24)}$ used the sensitivity of polarized SAR signals in different time phases to classify land cover in urban areas in combination with backscattering intensity and coherence characteristics. Wu et al. ${ }^{(25)}$ used six images of ENVISAT ASAR with a time span of 4 years to analyze the typical coherent features in the coastal area of central Jiangsu at different spatial scales. The results showed that a change in spatial scale has a significant impact on the coherence for small-area features. However, the impact is small for large-area features. Many scholars have analyzed the factors that affect the coherence of SAR images from the perspective of sensor system design and seasonal variation, and have made some achievements. However, when SAR image coherence has been applied to detecting urban changes, most studies did not consider the difference among different ground objects in urban areas. Wu et al. ${ }^{(25)}$ analyzed the impact of spatial scale changes on the coherence of typical features in urban areas, but they did not consider the impact of time on the coherence of features.

The aim of this study is to reveal the changes in the coherence of ground objects in urban areas over a year. The data of $29 \mathrm{C}$-band radar images of the European Space Agency Sentinel-1A satellite in 2018 were collected and used for the study. We used the technique of differential synthetic aperture radar interferometry (DInSAR) to analyze the coherence changes of five typical ground features in urban Beijing and considered the effect of the weather on the coherence. Additionally, the reasons for the changes in the coherence of vegetation and bare soil are systematically discussed. In Sect. 2, we briefly introduce the study area and experimental data. Section 3 describes in detail the method of coherence analysis for different ground features in Beijing over one year. In Sect. 4, we discuss and analyze the differences in coherence obtained for the different ground features. Section 5 gives the conclusions of the study. 


\section{Study Area and Experimental Data}

\subsection{Study area}

In this study, urban Beijing was selected as the research area, where the region of interest was $115.7-117.4^{\circ} \mathrm{E}, 39.4-41.6^{\circ} \mathrm{N}$. The total area of Beijing is $16410 \mathrm{~km}^{2}$. In 2018 , the forest greening rate was $61.5 \%$. The terrain of the city is high in the northwest and low in the southeast. The city belongs to the warm temperate zone with a semi-humid and semi-arid monsoon climate. Among the different types of urban coverage, five typical ground features were selected in the plain area to explore their changes in coherence over one year: residential areas, vegetation, bare soil (covered with dust-proof green nets), bridges, and factories and warehouses, which are shown in Fig. 1.

\subsection{Experimental data}

To obtain the temporal changes in the coherence of the different ground features in the study area, 29 Sentinel-1A ascending images acquired by a SAR sensor were selected as the data source. The data were acquired from January to December 2018. The imaging band was the C-band $(5.6 \mathrm{~cm})$, the antenna transmission and reception were both vertical polarization (VV), the incident angle of the radar wave at the center of the images was $38.9^{\circ}$, the SAR image spatial

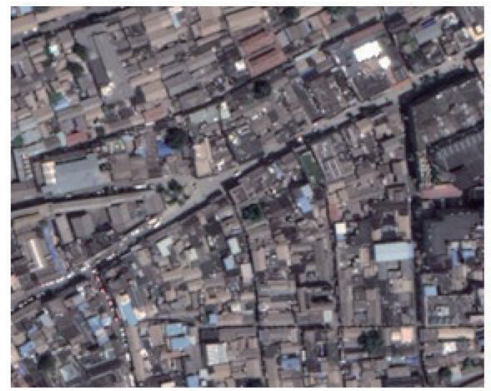

(a)

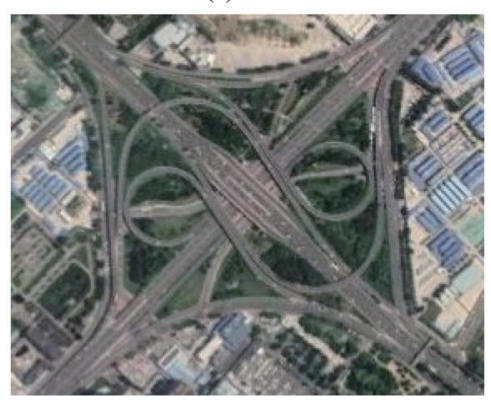

(d)

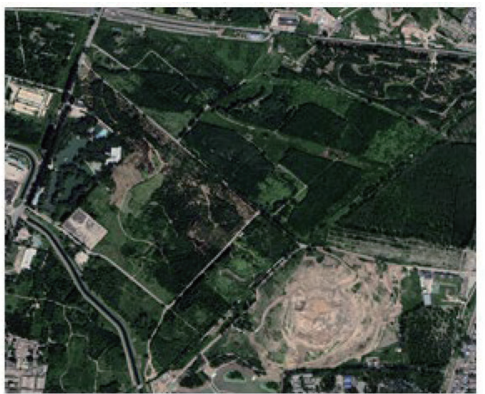

(b)

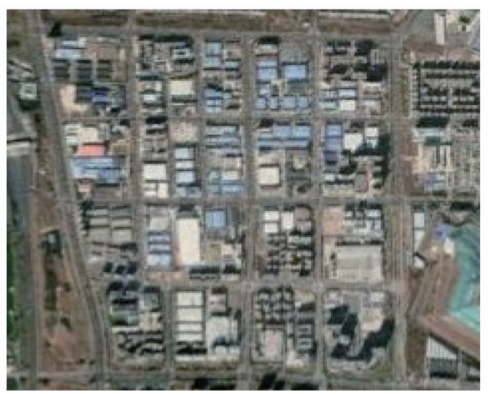

(e)

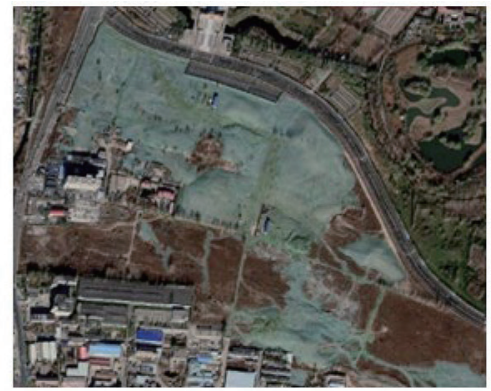

(c)

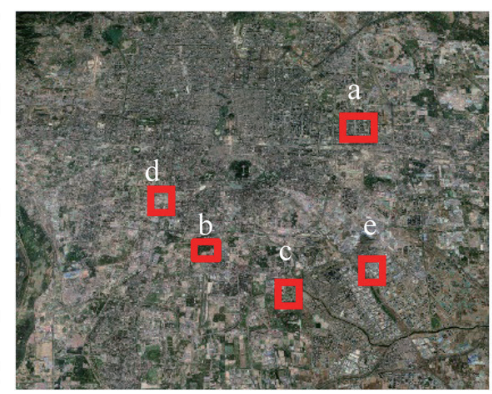

(f)

Fig. 1. (Color online) Five typical ground features: (a) residential areas, (b) vegetation, (c) bare soil (covered with dust-proof green nets), (d) bridges, and (e) factories and warehouses. (f) Distribution of different features in study area (specific locations of five typical ground features have been indicated). 
resolution was $5 \times 20 \mathrm{~m}^{2}$ (range direction $\times$ azimuth), and the ground coverage of each image was about $250 \times 250 \mathrm{~km}^{2}$. The external DEM was Shuttle Radar Topography Mission (SRTM) data with a resolution of $90 \mathrm{~m}$, which was used to remove the topography phase effect. DInSAR was performed on the acquired adjacent images, and a total of 28 pairs of interference images were acquired.

\section{Ground Object Coherence Measurement and Data Processing}

\subsection{Coherence coefficient of ground features}

SAR is a kind of radar, and coherence is one of the key factors used to ensure the azimuth imaging performance. An important indicator of coherence is the coherence coefficient. Generally, for two random variables with complex Gaussian distributions $z_{1}$ and $z_{2}$, their normalized complex coherence coefficient can be expressed as

$$
\gamma=\frac{\left.<z_{1} z_{2}^{*}\right\rangle}{\sqrt{\left\langle z_{1} z_{1}^{*}><z_{2} z_{2}^{*}\right\rangle}},
$$

where $<>$ represents the expected value and * represents the complex conjugate. If the position and backscattering coefficient of a single scatterer in the resolution unit are the same in two SAR images and the radar viewing direction remains unchanged, then the echo of the resolution unit will remain unchanged. For the pixels of two SAR complex images, $\gamma=0$ means complete decoherence, $\gamma=1$ means complete coherence, and $0<\gamma<1$ means partial coherence. ${ }^{(26)}$

In an actual estimation, the method of window estimation is used to calculate the coherence coefficient of two SAR images. The formula used is ${ }^{(27)}$

$$
\hat{\gamma}=\frac{\sum_{n=1}^{N} z_{1 n} z_{2 n}^{*}}{\sqrt{\sum_{n=1}^{N}\left|z_{1 n}\right|^{2}} \sqrt{\sum_{n=1}^{N}\left|z_{2 n}\right|^{2}}},
$$

where each summation is over the $N$ adjacent pixels around the center pixel.

\subsection{Time series DInSAR processing}

The choice of the SAR interferometric image pair mainly requires the consideration of the appropriate time and space baselines. The larger the time baseline, the lower the coherence; the longer the spatial baseline, the greater the decoherence. To study the temporal changes in the coherence of different features, it is necessary to control the spatial baseline within a certain range so that it does not exceed a certain threshold, so as to eliminate the effect of the spatial baseline on the coherence of different features. In this study, the absolute value of the maximum vertical baseline is $120 \mathrm{~m}$, and the 28 sets of processed interference pairs meet the above conditions, as shown in Table 1. 
Table 1

Basic parameters of interference image pairs.

\begin{tabular}{|c|c|c|c|c|}
\hline Serial number & Master & Slave & Time baseline & Vertical baseline \\
\hline 1 & 20180103 & 20180115 & 12 & 54 \\
\hline 2 & 20180115 & 20180127 & 12 & 50 \\
\hline 3 & 20180127 & 20180208 & 12 & 57 \\
\hline 4 & 20180208 & 20180220 & 12 & 10 \\
\hline 5 & 20180220 & 20180304 & 12 & 19 \\
\hline 6 & 20180304 & 20180328 & 24 & 31 \\
\hline 7 & 20180328 & 20180409 & 12 & 39 \\
\hline 8 & 20180409 & 20180421 & 12 & 13 \\
\hline 9 & 20180421 & 20180503 & 12 & 68 \\
\hline 10 & 20180503 & 20180515 & 12 & 49 \\
\hline 11 & 20180515 & 20180527 & 12 & 31 \\
\hline 12 & 20180527 & 20180608 & 12 & 10 \\
\hline 13 & 20180608 & 20180620 & 12 & 30 \\
\hline 14 & 20180620 & 20180702 & 12 & 57 \\
\hline 15 & 20180702 & 20180714 & 12 & 7 \\
\hline 16 & 20180714 & 20180726 & 12 & 20 \\
\hline 17 & 20180726 & 20180807 & 12 & 42 \\
\hline 18 & 20180807 & 20180819 & 12 & 65 \\
\hline 19 & 20180819 & 20180831 & 12 & 33 \\
\hline 20 & 20180831 & 20180912 & 12 & 58 \\
\hline 21 & 20180912 & 20180924 & 12 & 36 \\
\hline 22 & 20180924 & 20181006 & 12 & 24 \\
\hline 23 & 20181006 & 20181018 & 12 & 114 \\
\hline 24 & 20181018 & 20181111 & 24 & 110 \\
\hline 25 & 20181111 & 20181123 & 12 & 69 \\
\hline 26 & 20181123 & 20181205 & 12 & 37 \\
\hline 27 & 20181205 & 20181217 & 12 & 25 \\
\hline 28 & 20181217 & 20181229 & 12 & 94 \\
\hline
\end{tabular}

Because the Sentinel-1A satellite has high orbital accuracy and adopts the Terrain Observation by Progressive Scans (TOPS) mode imaging technology, the spatial baseline of the obtained interference pair is mostly below $70 \mathrm{~m}$ and the maximum spatial baseline is $114 \mathrm{~m}$, so the effect of the spatial baseline on the coherence can be ignored. To obtain the changes in coherence in the time series of typical features in the study area, 28 sets of time series differential interferograms and corresponding coherence coefficient maps between adjacent images of residential areas, vegetation, bare soil, bridges, and factories and warehouses were obtained. The coherence coefficient maps were used to analyze the changes in the coherence of the five types of ground features in the urban area over one year. The specific processing steps are as follows:

(1) The method of image cross-correlation was employed for coarse registration. Of the adjacent image pairs, the one with the earlier imaging date is the master image. For example, for the interference pair 20180103-20180115, the image with the date of 20180103 is the master image and the other image is the slave image. The registration accuracy can reach 0.01 pixel in the azimuth direction. Even though such a high accuracy was achieved, owing to the 
change in the Doppler centroid in different bursts, phase discontinuity will still occur in the azimuth direction. Therefore, it is necessary to accurately register each image.

(2) The offset of low-order terms between images was obtained by enhanced spectral diversity for fine registration. In this algorithm, the phase consistency of the overlapped regions between adjacent bursts was considered as the iterative standard to eliminate the phase discontinuity in the overlapping regions. Thus far, the image data of the top mode have been accurately registered, and the registration accuracy could meet the requirements of the subsequent interferogram generation.

(3) After the effects of the terrain and ground phases were removed by using orbit data and the reference DEM, the differential interferograms and master-slave image intensity maps were obtained.

(4) To improve the definition of interference fringes and reduce the decoherent noise caused by the spatial baseline, the 28 interference pairs were processed by Goldstein filtering, the coherence of each interference pair was calculated, and finally the filtered interference and coherence coefficient maps were obtained.

(5) Targeting the above five typical ground features, the changes in coherence over time were analyzed.

\section{Analysis of Coherence of Typical Features over Time}

\subsection{Test results of coherence of typical objects}

After performing differential interference processing 28 times for the five typical features, 140 coherence coefficient maps were obtained. Because of the limited space available, only the coherence coefficient maps and histograms of the different surface features in the imaging period from 20180819 to 20180831 were selected for a brief analysis. Figure 2(a) shows the coherence coefficient diagrams of typical ground features and Fig. 2(b) shows the corresponding histograms.

It can be seen from Fig. 2 that in the period of 20180819-20180831, the coherence coefficient of residential areas was mainly distributed in the range [0.5, 0.9], and the corresponding ranges for vegetation, bare soil, bridges, and factories and warehouses were [0, 0.4], [0.1, 0.6], [0.4, 0.95], and [0.6, 0.95], respectively.

Comparing the coherence coefficient maps and histograms of the different ground features, we found that if the coherence coefficient map of the ground object is bright, the pixel values in the corresponding histogram are mainly distributed in the range of $[0.5,1]$, which proves that the ground object has good coherence in the SAR image; in contrast, if the coherence coefficient map of the ground object is dark, the pixel values in the corresponding histogram are mainly distributed in the range of $[0,0.5]$. In addition, the histograms for vegetation and bare soil are similar. 


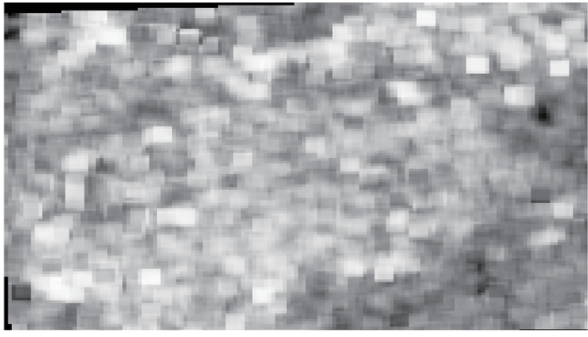

(a1)

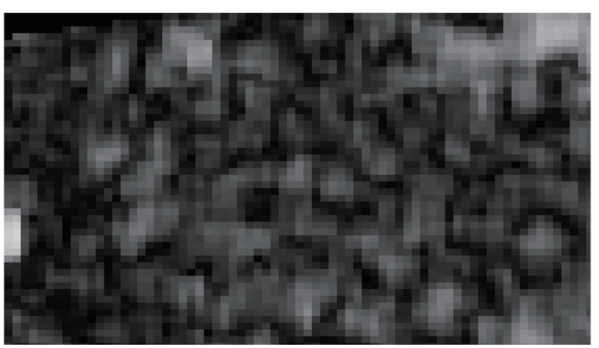

(a2)

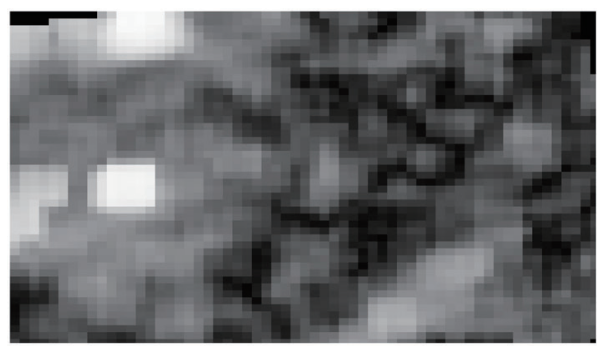

(a3)

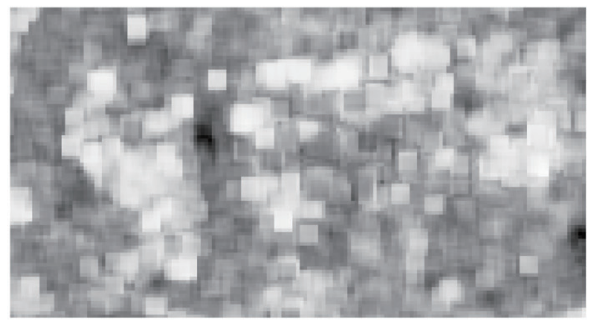

(a4)

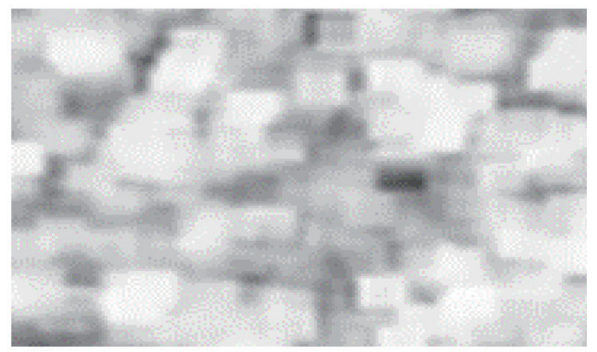

(a5)

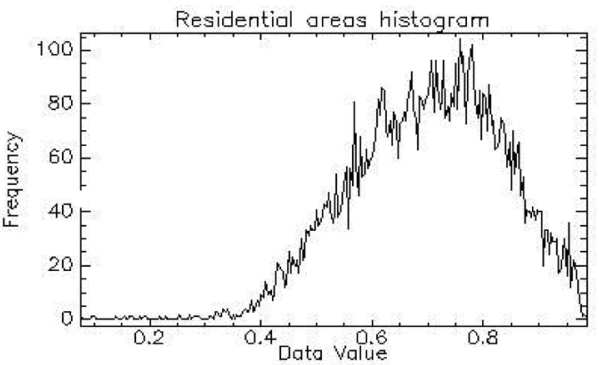

(b1)

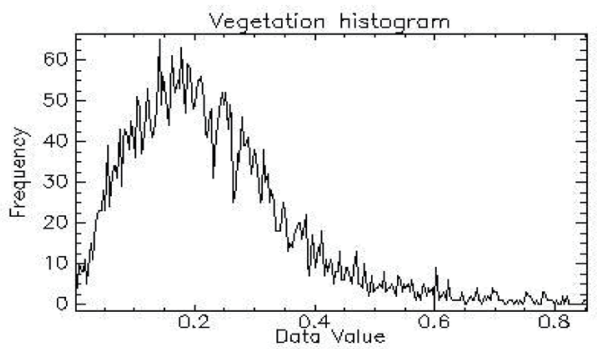

(b2)

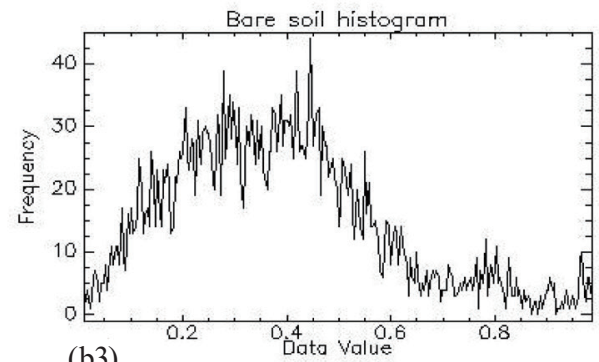

(b3)
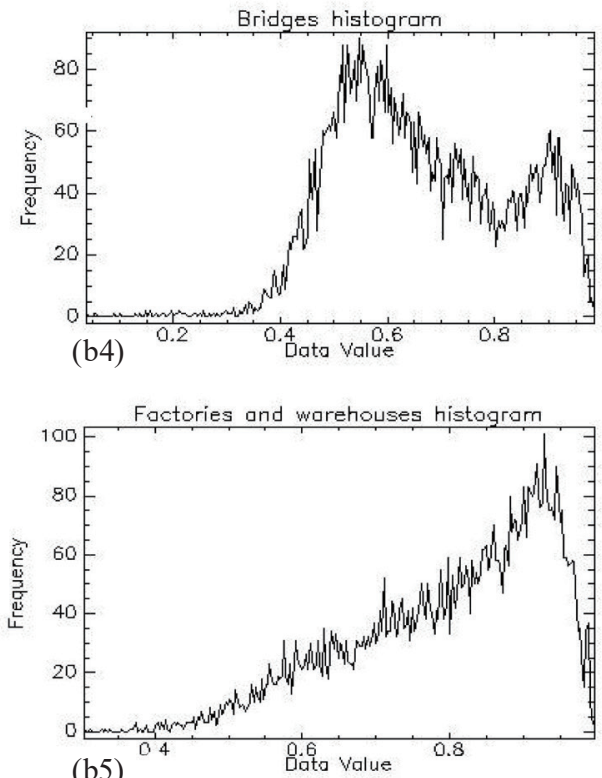

(b5)

Fig. 2. Coherence coefficient maps and histograms of different typical features. (a1) and (b1) Coherence coefficient map and histogram of residential areas, respectively. (a2)-(a5) and (b2)-(b5) Coherence coefficient maps and histograms of vegetation, bare soil, bridges, and factories and warehouses, respectively. 


\subsection{Analysis of the coherence of typical features over time}

The coherence coefficients of the five ground features obtained by DInSAR over one year are plotted in Fig. 3. It can be seen from Fig. 3 that the coherence is highest for factories and warehouses over the year, with maximum, minimum, and average values of $0.86,0.57$, and 0.75 , respectively. However, the volatility from June to August is relatively high; possibly vegetation in the grounds of factories and warehouses grows luxuriantly during the summer, which affects the coherence characteristics of the area. The coherence of residential areas and bridges is relatively consistent over the year, with a lower coefficient for bridges. The maximum coherence coefficient of residential areas is 0.78 , the minimum value is 0.65 , and the average value is 0.72 , while the corresponding values for bridges are $0.76,0.57$, and 0.67 , respectively. The coherence coefficient of bare soil is low, with a maximum value of 0.78 , a minimum value of 0.39 , and an average value of 0.63 , and decreases from January to August then steadily increases from September to December. The coherence coefficient of vegetation is the lowest, with maximum, minimum, and average values of $0.68,0.23$, and 0.46 , respectively.

From the above analysis, we found that the artificial ground features (factories and warehouses, residential areas, and bridges) maintained high backscattering characteristics over one year and the coherence coefficient was stable, while natural ground features (bare soil and vegetation) showed low backscattering characteristics and the coherence coefficient fluctuated greatly.

A line chart of the coherence coefficients for bare soil and vegetation is shown in Fig. 4. It can be seen that the coherence coefficients of vegetation and bare soil are relatively stable from January 3 to April 9 then drop sharply from April 9 to April 21, before rising sharply from May 3 to May 15. After that, the coherence coefficients decrease approximately linearly, then increase again from August 31 to September 12, after which they increase approximately

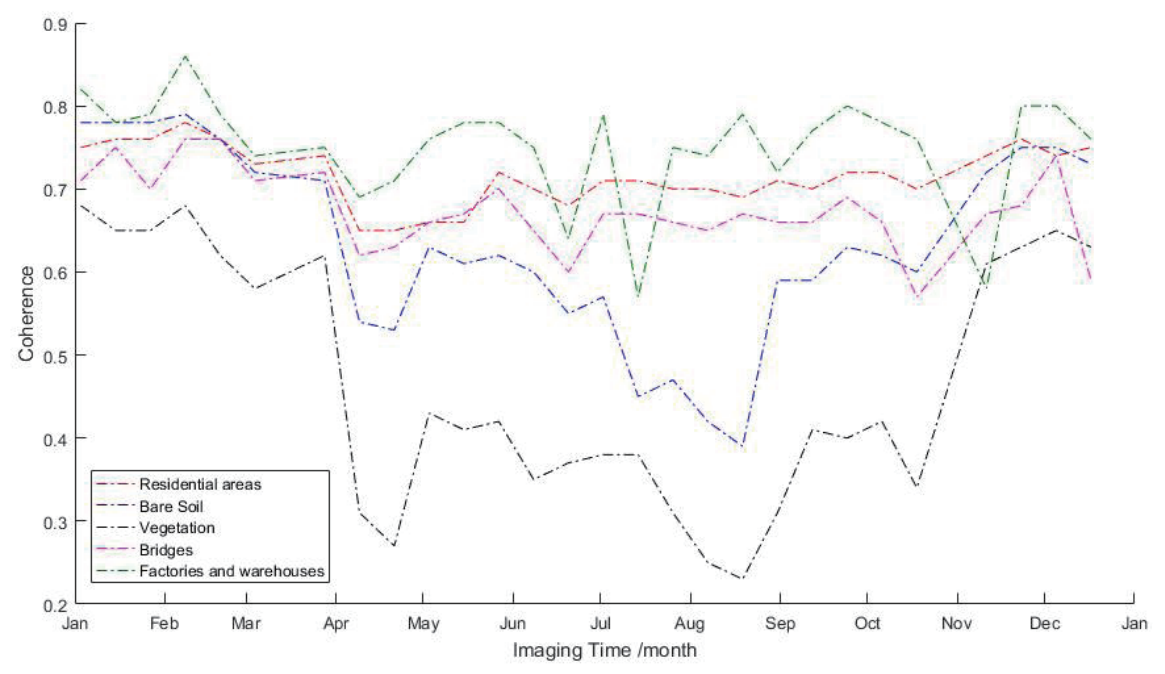

Fig. 3. (Color online) Line chart of changes in coherence of ground features. 


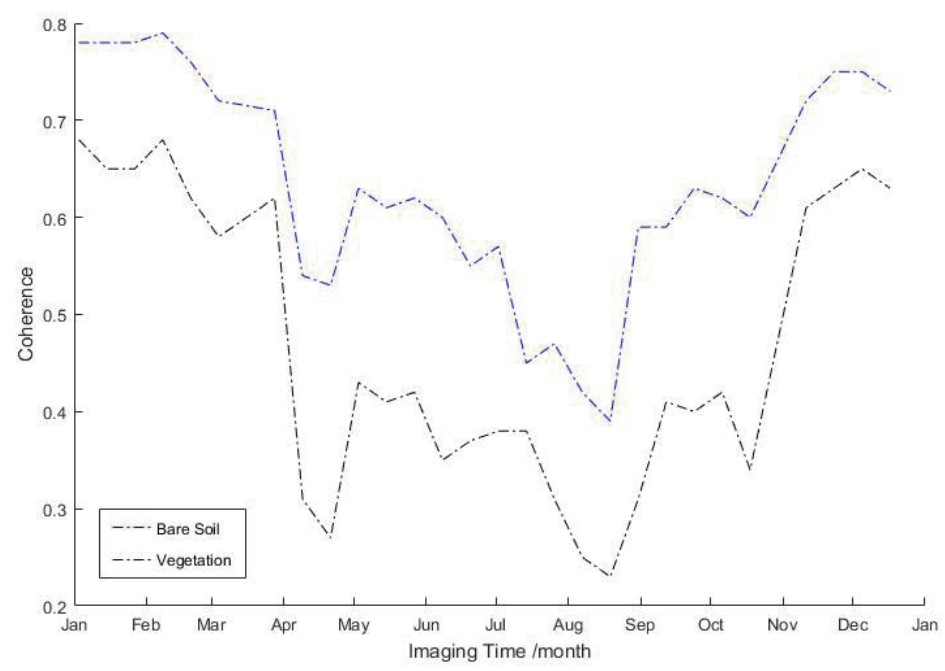

Fig. 4. (Color online) Coherence variations of vegetation and bare soil.

linearly. A strong correlation was found in the coherence changes of bare soil and vegetation. To explore the reason for this, a further analysis of the coherence coefficients of bare soil and vegetation was carried out.

According to the research results of Kubica et al. ${ }^{(28)}$ and Bai et al.. ${ }^{(29)}$ the coherence of ground objects is affected by the time interval of SAR images, the wavelength parameters, and the properties of the ground objects, such as the dielectric constant, surface roughness, and backscattering intensity. In this experiment, Sentinel-1A data were used for interferometric processing, while the time and spatial baselines did not exceed a certain threshold, so their impact on coherence can be ignored. The wavelength parameters were determined by the radar satellite system parameters. Therefore, only the change in dielectric constant caused by the state of the ground object and the atmospheric conditions, and the effect of time decoherence caused by changes in weather were considered here.

By querying the meteorological data of Beijing in 2018 (http://tianqi.2345.com/wea history/54511.htm), we found that from April 4 to April 21, there were thirteen days of cloudy and rainy weather.

There are three possible reasons for the sudden drop in the coherence of vegetation and bare soil from April 9 to April 21. (1) The weather varied during this period, reducing the coherence through atmospheric phase noise. (2) This period was dominated by cloudy and rainy days accompanied by a strong wind, causing the ground features of vegetation and bare soil to rapidly change. (3) The dielectric constant of soil was changed by rainfall, enhancing the backscattering coefficient and reducing the coherence of ground objects.

There are two possible reasons for the enhanced coherence of vegetation and bare soil from May 3 to May 15. (1) During the imaging period, the weather was relatively stable, that is, mainly sunny with no strong wind. Therefore, the morphology and structure of vegetation and bare soil were relatively stable. (2) The sunny weather restored the original dielectric constant of the soil, so the backscattering intensity was relatively stable and the coherence was improved. 
From May 15 to August 31, the coherence coefficients of vegetation and bare soil linearly decreased, which was mainly related to seasonal factors. In summer, the morphology and structure of vegetation and bare soil were easily affected by external environmental factors, so the coherence tended to decrease.

From August 31 to September 12, the coherence coefficients of vegetation and bare soil increased again. There are two possible reasons for this. (1) The atmospheric conditions were relatively stable during this period, so the dielectric constant was stable. (2) This period was in autumn and plants grew slowly, so the terrain was relatively stable. In addition, the effects of external environmental factors were small, thus improving the coherence.

From September 12 to December 29 (autumn and early winter), the morphology and structure of the vegetation and bare soil were stable with little effects from external environmental factors. Therefore, the coherence increased approximately linearly, then tended to be stable from January 3 to April 9 (winter and early spring).

\section{Discussion and Conclusions}

The coherence coefficient is a very important parameter in radar interferometry, and it can directly reflect the characteristics of surface changes. In this paper, taking into the urban area of Beijing as the research object, 29 Sentinel-1A images acquired by a SAR sensor were used to analyze the changes in the coherence of typical features in the area over one year. The research results are as follows:

(1) The coherence of artificial features was well maintained over the year. Among them, residential areas had the most stable coherence, with a coherence coefficient variation of 0.13 over the year, and their scattering characteristics were not easily affected by external environmental variables. In contrast, the coherence of natural features (vegetation and bare soil) fluctuated greatly over the year, with the coherence coefficient ranging by as much as 0.45 . Their scattering characteristics were easily affected by changes in dielectric constant caused by the state of the features and atmospheric conditions. The coherence of natural features was low.

(2) It is of practical value to study the temporal changes in the coherence of different ground features. For example, the results can be used for the rough classification of land into different types and the effective selection of points with high coherence in SAR images. In this paper, the variations in the coherence coefficients of vegetation and bare soil over a year were analyzed by considering the state of the ground surface, the dielectric constant, and changes in the weather, but the mechanism causing the changes in coherence was not discussed in depth and should be studied in the future.

(3) In general, the coherence of different ground objects will be affected by changes in weather. In spring (March to May) and summer (June to August), the coherence of different ground objects tended to decrease and fluctuate greatly, whereas the coherence in autumn (September to November) and winter (December to February) tended to increase and the coherence from December to February was relatively stable. 


\section{Acknowledgments}

This study was sponsored by the National Natural Science Foundation of China (grant no. 41871367), the Ministry of Science and Technology of the People's Republic of China (grant no. 2018YFE0206100), the Importation and Development of High-Caliber Talents Project of Beijing Municipal Institutions (grant no. CIT\&TCD201704053), the Science and Technology Project of Ministry of Housing and Urban-Rural Development of the People's Republic of China (grant no. 2017-K4-002), the Scientific Research Project of Beijing Educational Committee (grant no. KM201910016007), the Major Projects of Beijing Advanced Innovation Center for Future Urban Design (grant no. UDC2018031321), and the BUCEA Postgraduate Innovation Project.

\section{References}

1 A. Elmzoughi, R. Abdelfattah, and Z. Belhadj: Proc. 2009 16th IEEE Int. Conf. Image Processing (2009) 1337.

2 L. Ai, L. Pang, H. Liu, M. Sun, and S. He: Proc. Int. Conf. Geo-Informatics in Resource Management and Sustainable Ecosystem. (2015) 464.

3 D. Xiang, T. Tang, Y. Ban, Y. Su, and G. Kuang: Appl. Geophys. 116 (2016) 86. https://doi.org/10.1016/ j.isprsjprs.2016.03.009

4 P. Berardino, G. Fornaro, R. Lanari, and E. Sansosti: IEEE Trans. Geosci. Remote Sens. 40 (2002) 2375. https://doi.org/10.1109/TGRS.2002.803792

5 M. Pietro., G. Giorgia, D. Matthew, P. Daniele, and M. Giovanni: Remote Sens. 10 (2018) 287. https://https:// doi.org/10.3390/rs10020287

6 D. Perissin, Z. Wang, and H. Lin: ISPRS J. Photogramm. Remote Sens. 73 (2012) 58. https://doi.org/10.1016/ j.isprsjprs.2012.07.002

7 S. Dong, S. Samsonov, H. Yin, S. Ye, and Y. Cao: Environ. Earth Sci. 72 (2014) 677. https://doi.org/10.1007/ s12665-013-2990-y

8 M. Chini., R. Pelich, L. Pulvirenti, and N. Pierdicca: Remote Sens. 11 (2019) 1. https://doi.org/10.3390/ rs11020107

9 C. H. Lu, C. F. Ni, C. P. Chang, J. Y. Yen, and R. Y. Chuang: Remote Sens. 10 (2019) 1318. https://doi. org/10.3390/rs10081318

10 V. Perumal: J. Appl. Remote Sens. 7 (2013) 1. https://doi.org/10.1117/1.JRS.7.073592

11 K. Hachem, M. Jurchescu, F. Grecu, A. Ozer, and M. Visan: Adv. Remote Sens. 3 (2014) 23. https://doi. org/10.4236/ars.2014.31003

12 J. Hagberg, L. Ulander, and J. Askne: IEEE Trans. Geosci. Remote Sens. 33 (1995) 331. https://doi. org $/ 10.1109 / 36.377933$

13 A. Novellino, F. Cigna, M. Brahmi, A. Sowter, L. Bateson, and S. Marsh: Geosci. J. 7 (2017) 19. https://doi. org/10.3390/geosciences7020019

14 M. Watanabe, R. Bahador, T. Ohsumi, H. Fujiwara, C. Yonezawa, N. Tomii, and S. Suzuki: Earth Planets Space. 68 (2016) 131. https://doi.org/10.1186/s40623-016-0513-2

15 J. DeLaurentis: IET Radar Sonar Navig. 5 (2011) 561. https://doi.org/10.1049/iet-rsn.2010.0225

16 C. Xie, Z. Li, and X. Li: J. Glaciol. Geocryol. 30 (2008) 868. https://doi.org/1000-0240(2008)05-0868-07

17 C. Ichoku, A. Karnieli, Y. Arkin, J. Chorowicz, T. Fleury, and J. Rudant: Int. J. Remote Sens. 19 (2010) 1147. https://doi.org/10.1080/014311698215658

18 D. Andre and K. Morrison: Proc. 2016 11th European Conf. Synthetic Aperture Radar (2016) 1187.

19 X. Lv, M. Xing, Y. Deng, S. Zhang, and Y. Wu: IEEE Trans. Geosci. Remote Sens. 47 (2009) 2884. https://doi. org/10.1109/TGRS.2009.2018532

20 B. Anura, D. Michael, H. Stephen, A. Stefan, E. Jonas, and L. Brad: Int. J. Remote Sens. 37 (2016) 370. https:// doi.org/10.1080/01431161.2014.915435

21 Z. Chang, W. Zhao, R. Yang, and T. Xue: Proc. 2012 2nd Int. Conf. Remote Sensing, Environment and Transportation Engineering (2012) 1.

22 X. Zhang, W. Liu, and S. He: J. Eng. Sci. Technol. Rev. 11 (2018) 18. https://doi.org/10.25103/jestr.113.03

23 L. Pulvirenti, M. Chini, N. Pierdicca, and G. Boni: IEEE Trans. Geosci. Remote Sens. 54 (2016) 1532. https:// doi.org/10.1109/TGRS.2015.2482001 
24 X. Wang, C. Erxue, Z. Li, and W. Yao: Acta Geod. Cartogr. Sin. 44 (2015) 533. https://doi.org/10.11947/ j.AGCS.2015.20130244

25 H. Wu, H. Zhang, C. Wang, and Y. Tang: Remote Sens. Technol. Appli. 24 (2009) 109. https://doi.org/10.11873/ j.issn.1004-0323.2009.1.109

26 T. Wu, H. Zhang, C. Wang, Y. Tang, and H. Wu: Chin. Sci. Bull. 53 (2008) 3705. https://doi.org/10.1007/ s11434-008-0331-4

27 R Touzi, A. Lopes, J. Brunique, and P. Vachon: IEEE Trans. Geosci. Remote Sens. 37 (1999) 135. https://doi. $\operatorname{org} / 10.1109 / 36.739146$

28 V. Kubica, X. Neyt, and H. Griffiths: IEEE Trans. Aerosp. Electron. Syst. 52 (2016) 1568. https://doi. org/10.1109/TAES.2016.140831

29 Z. Bai, S. Fang, J. Gao, Y. Zhang, G. Jin, S. Wang, Y. Zhu, and J. Xu: Sci. Rep. 1 (2020) 1. https://doi. org/10.1038/s41598-020-63560-0

\section{About the Authors}

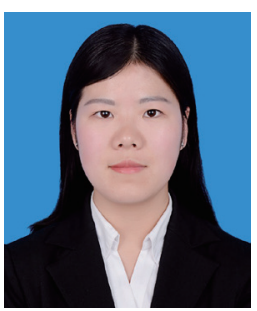

Jiaojie Li received her B.S. degree in surveying and mapping engineering from North China University of Water Resources and Electric Power in 2019. She is currently a graduate student in photogrammetry and remote sensing at Beijing University of Civil Engineering and Architecture. Her research interests include the monitoring of coseismic deformation fields based on DInSAR technology. (2108160219002@stu.bucea.edu.cn)

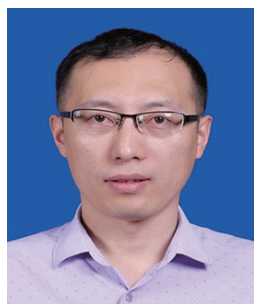

Xuedong Zhang is an associate professor and master tutor of Beijing University of Civil Engineering and Architecture. He graduated from China University of Mining and Technology (Beijing) in 2012 with a doctorate degree in engineering and is currently engaged in research on InSAR and urban remote sensing. He has published more than 30 related papers and won four provincial and ministerial awards. (zhangxuedong@bucea.edu.cn)

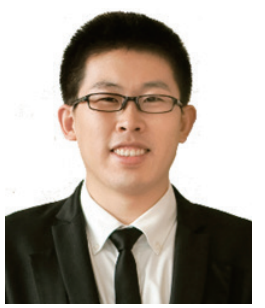

Xianglei Liu received his B.S. and M.S. degrees in geographic information systems from Shandong University of Science and Technology in 2005 and 2008, respectively. He received his Ph.D. degree in photogrammetry and remote sensing from Tongji University in 2012. He is a professor at Beijing University of Civil Engineering and Architecture. His research interests are in deformation monitoring based on GBSAR and high-speed videogrammetric measurement. (liuxianglei@bucea.edu.cn) 